\title{
Practical risk management in early phase clinical trials
}

\author{
Simon Coates ${ }^{1} \cdot$ Jörg Täubel ${ }^{1,2} \cdot$ Ulrike Lorch $^{1}$ \\ Received: 5 September 2018 / Accepted: 30 November 2018 / Published online: 19 December 2018 \\ (C) The Author(s) 2018
}

\begin{abstract}
Purpose Stopping rules are an essential part of risk management in early phase clinical trials. As well as being necessary for ensuring the safety of participants on clinical trials, they are also a requirement under the revision to the European Medicine Agency's first-in-human and early clinical trial guideline. The increasing complexity and size of modern trial designs (e.g. integrated trials) raise potential issues with risk management, which, if also too complex, presents challenges for both regulators and investigators to implement. Therefore, there is a clear need for a standard, template, or algorithm-based approach to risk management, in particular rules concerning adverse reactions. The purpose of this manuscript is to present template stopping (or adverse reaction, AR) rules that fulfil regulatory requirements and that can be adapted, taking into account trial design, nature of the investigational medicinal product, and anticipated effects.

Methods The template AR rules that use a systematic, objective and consistent process were developed, taking into account severity (using an objective grading system), seriousness, frequency and reversibility of ARs. These rules control decisions relating to individual trial participants, dosing regimens and dose escalation and/or progression to successive trial parts. For ease of use, the template rules consist of a single, one-page table.

Results The template AR rules have been successfully applied to many early phase adaptive integrated trials that received regulatory authorisation and were performed in the UK. This manuscript presents the template rule table and case studies of some trial-specific adaptations.

Conclusions This work demonstrates how a systematic, objective and consistent approach to risk management of large integrated trials can be simple yet robust, facilitating effective decision making and trial progression whilst safeguarding participant safety.
\end{abstract}

Keywords Risk management $\cdot$ Safety $\cdot$ Stopping rules $\cdot$ Adverse reactions $\cdot$ Clinical trials

\section{Introduction}

\section{Terminology used in this manuscript}

Adverse event (AE): any untoward medical occurrence in a subject to whom a medicinal product is administered and which does not necessarily have a causal relationship with this treatment [1-3].

Serious adverse event (SAE): any untoward medical occurrence or effect that at any dose results in death, is

Simon Coates

s.coates@richmondpharmacology.com

1 Richmond Pharmacology, St George's University, Cranmer Terrace, London SW17 0RE, UK

2 Cardiovascular and Cell Sciences Institute, St George's University, Cranmer Terrace, London, UK life-threatening, requires hospitalisation or prolongation of existing hospitalisation, results in persistent or significant disability or incapacity or is a congenital anomaly or birth defect $[1,3]$.

Adverse reaction (AR): all untoward and unintended responses to an investigational medicinal product related to any dose administered [1]. An adverse reaction: adverse events considered as, at least, possibly related to the investigational medicinal product (IMP) administration [4, 5]. The latter term definition provides clearer instruction on the term 'related'.

Serious adverse reaction (SAR): a serious adverse event considered at least possibly related to the IMP administration [4]. Note: The International Council for Harmonisation of Technical Requirements for Pharmaceuticals for Human Use (ICH) uses the terms adverse drug reaction (ADR) and serious ADR, which are synonymous with AR and SAR. For the purposes of this manuscript, the term AR will be used for consistency with the most recent regulatory documents. 
The Reference Safety Information (RSI) is used for the assessment of the expectedness of all 'suspected' serious adverse reactions (SARs) that occur in clinical trials. The list of 'expected SARs' should be based on 'suspected' SARs that were previously observed and not on the basis of what might be anticipated from the pharmacological properties of a medicinal product or the compound class. An 'expected' SAR is therefore one that is listed in the RSI [5]. For first-in-human and other early phase clinical trials where no or limited human data is available, there are unlikely to be any 'expected' SARs. However, class effects, mode of action and potentially nonclinical in vivo data may predict potential ARs in humans. For this scenario, the term 'anticipated' will be used.

Unexpected SAR: a serious adverse reaction, the nature, severity or outcome of which is not consistent with the reference safety information [2]. Similarly, SUSAR: Suspected Unexpected Serious Adverse Reaction, i.e. any SAR that is not listed in the RSI [5].

\section{The clinical need for rules dealing with adverse reactions}

Traditionally, clinical trials have been required to contain 'stopping rules', which are essentially a set of criteria that specify when dosing an individual subject, cohort and/or trial should be suspended. They are usually based on the occurrence and number of severe and serious adverse reactions (ARs). Consequently, ARs are one of the factors that will determine whether a trial continues or gets suspended-' go/no-go' decisions - and so require careful consideration prior to trial initiation. Recent events in early phase clinical trials [6-8] in Europe have highlighted the importance of risk management strategies. Yet, despite being an essential part of risk management, rules regarding ARs continue to pose challenges for Investigators and are often poorly defined in trial protocols $[9,10]$.

Recent changes in clinical trial design [4] potentially present further challenges for risk management. Current practice favours integrated protocols which allow the conduct of entire early phase programmes from first-in-human to proof of concept within one protocol. A typical large, integrated trial design may have multiple study types (e.g. single and multiple ascending doses, food effect, ethnic comparator and proof of concept) conducted, often in parallel, as separate arms of the same trial. Conducting many types of studies within the same protocol and thus requiring only a single regulatory approval brings time and cost savings, which has contributed to their increased popularity. The rules concerning ARs that will govern such a trial design may need to accommodate multiple trial arms running in parallel, which may use different dosing regimens (and exposures) of the investigational medicinal product (IMP). Yet, they also need to be readily understandable, robust and facilitate effective decision-making, with participant safety the first and foremost concern. The increasing frequency of integrated trials being designed supports the use of a standard approach to creating such rules, which may consequently ensure a consistent standard of safety monitoring.

For a conventional, unintegrated protocol, such as an early phase trial with a single cohort, or a single ascending dose study only, simple, high-level rules may suffice. For example, in a study with only single ascending dose cohorts, one highlevel rule stating 'if two or more subjects experience an AR of moderate severity in a cohort, the remainder of that cohort should not be dosed and escalation to the cohorts with higher dose levels is suspended' (or similar) may be sufficient.

However, such simplistic, high level rules have limitations:

(1) Firstly, they are just stopping rules. For a larger combined integrated protocol with multiple study parts, more comprehensive rules should allow more options than just stopping - e.g. extending a cohort to gather further information, continuation with modified dosing regimens (e.g. dose levels with anticipated lower exposures). More comprehensive rules could therefore potentially allow concurrent study parts running in parallel to continue.

(2) Secondly, the revision to the European Medicine Agency (EMA)'s guideline on first-in-human/early phase trials [4] sets requirements for ARs that are severe or serious. However, for a first-in-human trial, where the safety profile of the drug is not established, all ARs, even those at lower severity grades, should be considered when making decisions on trial continuation. The emergence of a series of moderate or even non-serious severe ARs in a first-in-human trial may indicate a safety signal emerging.

(3) Thirdly, early phase trials, e.g. after the first year of development, where clinical safety data in the Investigator's Brochure (IB) or (if marketed) a Summary of Product Characteristics (SPC) is available, the drug may have documented 'anticipated effects' Additionally, pre-clinical data may suggest anticipated effects that may or may not be adverse. For these, a clear boundary needs to be defined between acceptable and unacceptable ARs (in terms of severity and/or numbers observed); defined, acceptable expected ARs should not trigger unnecessary or premature subject withdrawal or study suspension.

Therefore, simple high-level rules are unlikely to be suitable for larger integrated protocols. This manuscript aims to show template rules which were created for large integrated trials and how they can be adapted for different compounds and trial designs. The EMA guideline refers to these as stopping rules in case of severe or serious ARs. However, the template rules designed go beyond simply mandating 
stopping. They seek to deal with ARs of all severities as they arise and determine appropriate next steps. For this reason, the template rules are referred to as adverse reaction rules (AR rules).

\section{The regulatory requirement for AR rules}

As well as being essential for participant safety, AR rules are also a regulatory requirement and their use is stated in ICH 36 (R2) [3] and the Food and Drug Administration (FDA)'s Good Review Practice: clinical review of investigational new drug applications, FDA 2013 [11]. However, it is the EMA's latest revision to the first-in-human and early phase trial guideline [4] that addresses risk management in more complex modern trial designs, and it specifically addresses the need for clear rules for ARs. As well as stating that 'the protocol should define unambiguous stopping rules which result in an immediate stop to dosing', it also specifies a requirement to define stopping rules for each of the following:

- Final stop to dosing and termination of the trial;

- Stopping for an individual subject, at any time in the trial;

- Stopping within a cohort

- when subjects in a cohort are dosed staggered;

- during multiple dosing;

- Progression to the next part of the trial;

- Any dose escalation parts of the trial.

The template AR rules described in this paper have been designed to ensure they aid safe, effective Investigator and Safety Review Committee decision making, fulfil the requirements of the EMA's guideline and ensure regulator acceptability.

\section{Background to the authors and previous work}

All authors are both physicians and Investigators working in an early phase clinical trial unit. The authors have been developing systematic methods of designing and conducting clinical trials for several years. Previously published work included a retrospective analysis of time savings using adaptive design [12] and a later publication presented methodology for designing adaptive trials and their risk management [13]. The latter described a high-level approach to designing AR rules. During the 4 years since that publication, the Investigators have used that approach in numerous trials and created template AR rules that were adapted on a trial-by-trial basis, if appropriate. This manuscript presents our current template AR rules and examples of adaptations so that other researchers can use it.

\section{Methods}

\section{The assessment of ARs}

The template rules shown in this paper were designed for the most complex scenario as a starting point - a large integrated trial. The rules follow the assessment of ARs in clinical practice. In general, ARs are assessed in the following order:

Decision (1): The impact on the individual subject, e.g. whether the subject can continue to receive the IMP (therefore only applicable if they are due to receive multiple doses). This is determined using "individual AR rules".

Decision (2): The impact on the remainder of the cohort of which the affected subject is a part (i.e. the dosing regimen group). This applies in the following circumstances:

- If a cohort is split into different subgroups, such as sentinel cohorts, the impact on the successive subgroups of that cohort-i.e. whether the next successive subgroups can be dosed or not. This is applicable for single and multiple dosing regimens.

- If a cohort is due to receive multiple doses (e.g. a dose on the following day or week), whether that cohort can receive further doses as per the dosing schedule.

This is determined using "within-cohort AR rules".

Decision (3): The impact on:

- Escalation to cohorts with an expected higher exposure dosing regimen.

- Progression to successive parts of the study.

- Continuation or suspension of the overall study.

This is determined using "study progression AR rules".

The application of the above approach is shown diagrammatically in Fig. 1 which shows how the assessment of ARs needs to be made in a systematic order.

The template AR rules were created to reflect the three decisions, ensuring that the assessment of ARs occurs in a systematic order. All three decisions' rules were consolidated into a single table to facilitate ease of use and effective decision-making. The table contains the factors that need to be considered (input factors, described below), the numbers of subjects for each situation and the resulting outcome.

Investigators and sponsors therefore need to first determine which of the three decisions contained in the template AR rules table are needed, e.g. if the proposed trial is a single cohort only with no dose escalation or successive cohorts/ study parts, study progression rules may not be required. 


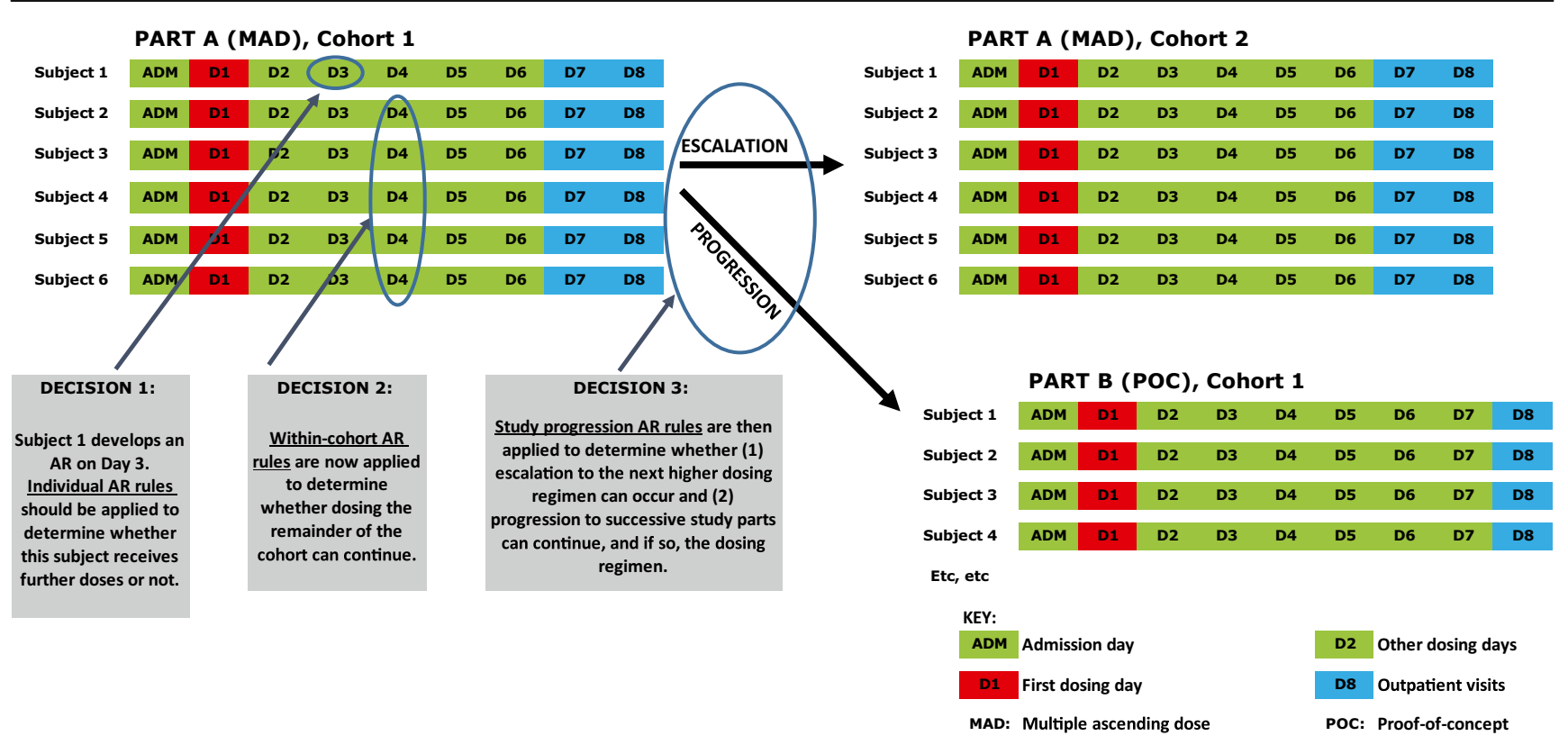

Fig. 1 The systematic assessment of ARs as a series of three decisions

Note that if the study does not contain dose escalation or progression to additional study parts, the within-cohort rules that determine when a cohort's dosing gets suspended will then essentially also function as the rules governing the overall study suspension.

\section{Designing the template AR rules: the input factors that were considered}

Terminology/grading system The severity of an AR will clearly be a major determining factor of a subject's continuation in a trial and the impact on the remainder of the trial. The use of an established scoring system is recommended as this will ensure that the assessment of AE severity is consistent and objective. Several AE grading systems are available, e.g. the National Cancer Institutes' Common Terminology Criteria for Adverse Events (CTCAE) [14], WHO Technical Report Series [11], the FDA Vaccine toxicity grading scale [15] and DAIDs AE grading table [16]. The CTCAE system was selected for the template AR rules as it is the most comprehensive reference document, it is based on "Medical Dictionary for Regulatory Activities" (MedDRA) [17] terminology and it is regularly updated. Additionally, its grading system (grades 1 to 5) aligns with the standard intensity grading of mild, moderate of severe used for adverse events during clinical studies: grade 1mild, grade 2-moderate, grade 3-severe, grade 4-life threatening and grade 5-death. Although the rules use CTCAE, most grading systems use a similar intensity scale for grading AEs, so one could be substituted for another (or the standard terms 'mild, moderate and severe' used) without changing the overall structure of the template rule table.
Predictability Broadly speaking, there are two categories of ARs:

- "Unanticipated"-ARs that could arise with any compound at any stage of drug development and the occurrence of these cannot be foreseen.

- "Anticipated"-the IMP's mode of action, pharmacodynamic effects, class effects and pre-clinical data may give an indication of potential or anticipated effects which should form the basis of risk management.

AR rules should balance the need to maintain subject safety whilst ensuring that the occurrence of mild or moderate anticipated effects does not trigger premature study termination. The same factors need to be considered for any active comparators/reference IMPs or non-IMPs (NIMPs).

When the template rules were adapted for certain IMPs (see discussion), any anticipated ARs and their potential severities were taken into account. Those that are potentially serious or life-threatening required more cautious rules, while those considered as evidence of drug efficacy and therefore potentially desirable drug effect were suitable for less cautious rules.

Severity (grade) and seriousness The severity and seriousness of ARs are the two variables that arguably have the biggest impact on the ability to continue dosing individuals and cohorts, and the ability to progress or escalate to other cohorts. In principle, as the severity increases, the number of subjects required to trigger suspension of dosing decreases.

The actions for grade 1 ARs are usually clear: Investigators would normally not stop a subject or any cohort dosing, even where there are many grade 1 ARs. Grades 3 to 5 (severe and/ 
or serious) are also clear and largely defined in the EMA's guideline [4] — one serious or two severe, non-serious ARs would normally mandate cessation in individual and cohort dosing and dose escalation. Grade 2 ARs are potentially more challenging as they are common but at moderate severity, a limit on acceptable numbers is arguably required.

Reversibility Reversibility is an important consideration when assessing ARs and the IMP's pharmacokinetics and the dosing schedule will determine the time period over when this should be assessed. For study progression decisions, there is likely to be a Safety Review Committee at the end of a cohort's dosing period before the next cohort or study part commences. This enables the reviewers to look at the entirety of the cohort's data and assess reversibility over longer periods of time before making the relevant decisions. For ongoing dosing in an individual or within a cohort, however, there may not be time to fully assess reversibility before the next dose is due so err on the side of caution. Ambiguous rules in a time-pressured environment are unhelpful and potentially dangerous, so rules need to be clear, but investigator judgement will always be needed. What constitutes reversibility is difficult to define but the decision should be made by an experienced clinician.

Numbers of subjects The rules are triggered by the number of subjects with one or more ARs, not the total number of ARs across all subjects. This is because the rules should be designed to capture emerging safety signals, which are more reliably identified by a trend, i.e. similar ARs in more than one subject (unless the events are serious). Three ARs in the same subject would not meet this objective - arguably, any subject who has experienced one side effect is more likely to experience others. Without additional subjects experiencing similar ARs, there is no clear trend that can be used to justify stopping dosing. In line with the principle that the rules should be applied when a potential safety signal has been identified, the number of subjects with ARs in the same SOC needs to be determined. A total number of subjects with ARs (i.e. not in the same SOC) that triggers an action should also be set, but this can be higher as different SOCs may not point to a definite safety signal.

The numbers for each AR (SOC and total) that trigger each action depend on the cohort size. Grade 1 would normally not trigger an action so no number needs to be set. For moderate (grade 2), the numbers need to be cautious enough that if a repeatable, clear (and potentially frequent) safety signal is identified, no further subjects are put at risk (if several grade 2 s have been observed in one cohort, escalating to a higher dose may result in grade 3 ARs emerging), but the numbers also need to avoid counter-productive and premature study suspension. The template rules have been used for cohort sizes of $4,6,8$ and 12 subjects and rules are triggered by three subjects (same SOC) and four (total, all SOCs) for grade 2 as they are sufficient to demonstrate a repeatable trend without being excessive. Consequently, these numbers could still be used for larger cohort sizes.

If reversibility is a built-in factor in the table, fewer subjects with grade 2 ARs would be needed to trigger suspension of dosing if they show no evidence of reversibility within the assessment period.

In line with the EMA's guidance, one subject with a serious AR or two subjects with severe, non-serious (grade 3 and above) ARs would suspend dosing in a cohort.

Actions For individual and within-cohort rules, it is important to keep the potential actions simple and clear as these decisions may need to be made by an Investigator or delegate prior to the next planned dosing. There may not be a scheduled Safety Review Committee meeting and the time in which to make a decision without interrupting dosing may be short. The rules should focus on the level at which an individual and cohort should have dosing suspended. For individuals, it is important to allow flexibility at grades 1 and 2 whereby the Investigator can use clinical judgement to decide whether a subject should be withdrawn at these lower grades.

For study progression rules, actions must clearly define the point at which the dosing of the entire study is suspended in line with the EMA guidance. For combinations of grades 1, 2 and 3 (non-serious) ARs, the rules can offer more options depending on the numbers seen. Continuation of the study as per protocol, extension of the cohort(s) to gather more data, continuation with lower exposure dosing regimens or continuation with only certain study parts/treatment arms are examples of such options that should be considered. To ensure regulatory acceptance, it is important that the rules state that trial or trial part continuation after rule-mandated suspension (even if only a temporary halt) will require substantial amendment to the protocol and competent authority approval.

\section{Results}

\section{Template AR rules}

Using the aforementioned methods, template AR rule tables were created in a consolidated table format, as shown in Table 1.

\section{Validation process}

All trials conducted by the authors have required rules concerning ARs. Their first publication [12] provided the approach being used in designing them. This approach has been refined over the succeeding years; the current template rules have been used since early 2016 and were presented at the European Federation for Exploratory Medicines Development (EUFEMED) conference in 2017 [18]. These template rules 


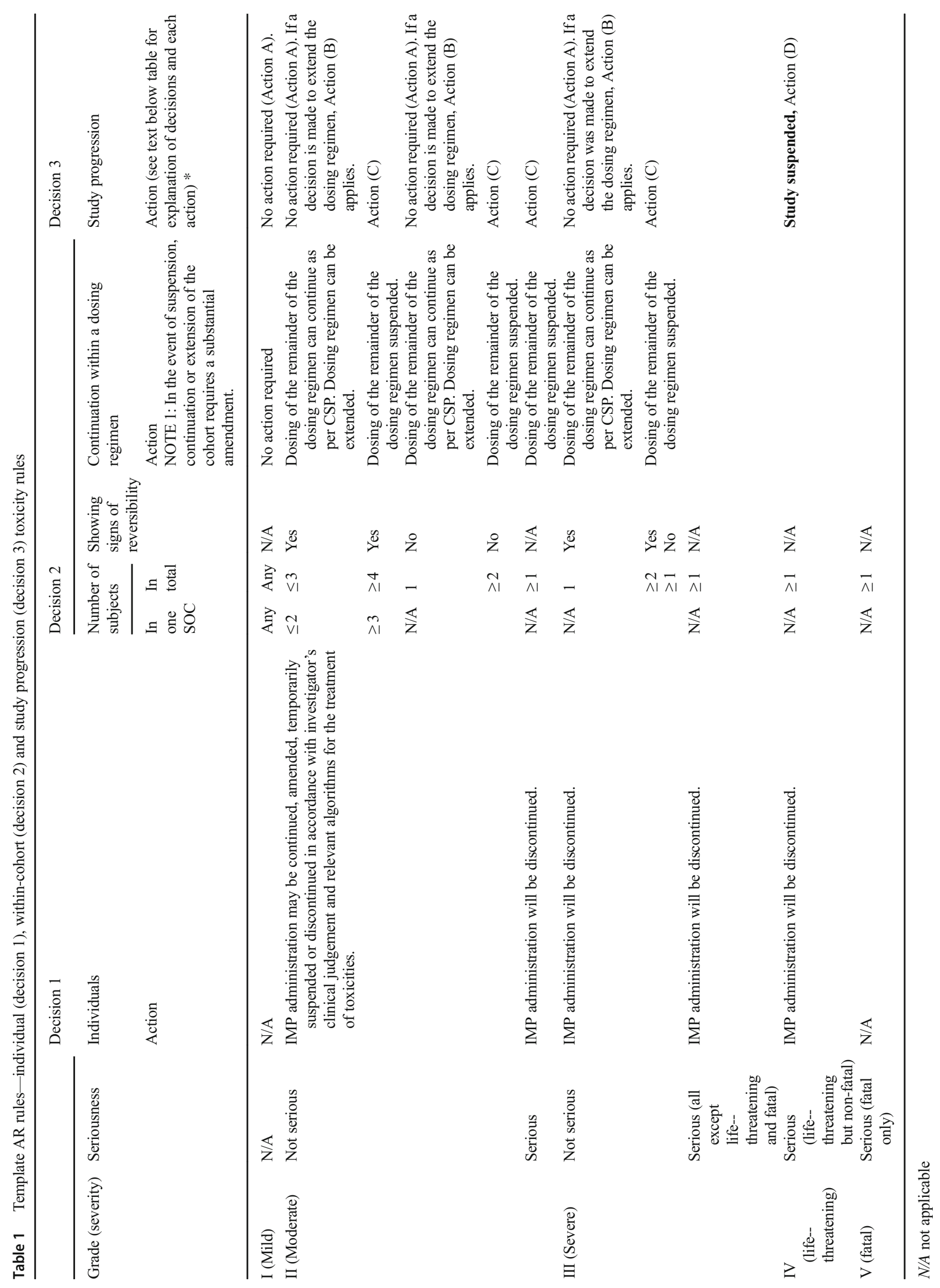


(or amended rules to suit the IMP or trial design) have been approved by competent authority (MHRA) for all first-inhuman and other early phase trials conducted at Richmond Pharmacology.

These template rules were first used in early 2016. The EMA's revision to the first-in-human guideline was released 10th November 2016. It utilised the same approach to AR rules already being used by the authors.

Although the rules themselves have not changed, they have since been consolidated into a single rules table for ease of use, which is designed shown in Table 1. This table has been approved and used in several early phase clinical trials since mid-2017. This consolidated rules table was presented at the 18th World Congress of Basic and Clinical Pharmacology (WCP) in Kyoto in 2018 [19].

\section{Discussion}

The template AR rules can be adapted to suit different trial designs and IMPs. This discussion will provide examples of such modifications and will use these to advise Investigators on how the AR rules can be implemented and modified as required for their own protocols. This discussion uses the examples to describe methodology and not the outcome of research in human subjects or data, therefore did not require Research Ethics Committee approval. Where a specific trial is used as an example, competent authority (Medicines and Healthcare Products Regulatory Agency, MHRA) and Research Ethics Committee approval were obtained for that trial and the EudraCT number will be stated.

The presence of anticipated or expected drug effects does not always warrant modification to the template tables. In the examples below, modifications were necessary either because a specific risk warranting stricter rules was identified or because drug effects were expected that were either desirable (evidence of pharmacodynamic effect) or did not pose a significant safety risk and consequently less strict rules were appropriate. If no specific risks are associated with predicted or expected effects, the AR rules can be used in their template form.

\section{Modifications for expected or anticipated effects based on product reference literature}

IMPs after the first year of development may have detailed RSI in the Investigator's Brochure (IB) detailing potentially serious expected drug effects. The IB (or Summary of Product Characteristics, SPC) may also contain information on nonserious ARs that have been observed and thus their appearance in later clinical trials can be anticipated. The frequency at which these ARs arise is understood - it is usually based on data from many clinical trials with a larger overall sample size 
(some SPCs state that the data is derived from hundreds or thousands of patients). The occurrence of these ARs in one or two subjects in a later clinical trial with a smaller number of subjects is less predictive than the IB/SPC of further individuals in the cohort being affected after continued dosing. Therefore, the rules can be kept simple-clear individual AR rules, in particular to define when a subject needs withdrawing, are essential. This may involve distinct rules for each significant AR listed in the IB or SPC. Within-cohort and study progression rules could then be based simply on the number of withdrawals.

For studies using active/reference IMPs/NIMPs where the SPC is the reference literature (e.g. in drug interaction studies), consider rules that allow suspension of one NIMP study part whilst continuing study parts with other NIMPs and/or the IMP. AR rules for the NIMP follow the same principles discussed above.

\section{Example 1}

A phase 1, drug-drug interaction study (EudraCT number 2016001958-18) tested the interactions of four NIMPs with the IMP in two separate, repeat-dose study parts (A and B). The IMP was a potent prostaglandin $\mathrm{F} 2 \alpha$ antagonist under development for pre-term labour. This trial evaluated any potential drug-drug interactions between the IMP and other standard-of-care medications. The part A NIMP (magnesium sulphate, administered in pre-term labour to prevent neurological defects in the infant) was expected to reach toxic levels and so the individual rules needed to be very strict (withdrawal at grade 1).
Individual rules (decision 1) Modified and split off from the template table and presented separately (see Table 2).

Within-cohort (decision 2) The template rules were deemed excessive as the IMP and NIMPs had known safety profiles (from the FIH trial and SPCs, respectively), so only simple rules to define the point at which dosing the cohort would stop were needed. These were based simply on the number of withdrawals. One SAR or two severe, non-serious ARs would stop the trial. If the SAR/ARs were due to one of the NIMPs, either that drug was removed from the trial, or if necessary that entire part would be discontinued.

Study progression (decision 3) Separate rules not requiredno dose escalation or progression to other study parts (parts A and B were parallel treatment arms only). Within-cohort rules functioned as study progression rules by default.

\section{Example 2}

A phase 1 study tested a new formulation of a marketed gynaecological drug which had an SPC. This was a single cohort study with healthy volunteers randomised to receive either placebo, a low strength or a higher strength formulation.

Individual rules (decision 1) Simplified, any subject with a safety concern (irrespective of CTCAE grade) was withdrawn. This was appropriate for this trial as the IMP was marketed with a satisfactory and well-established safety profile so the potential risks to individual safety to be monitored for were known at trial initiation.

Table 2 Individual AR rules, modified for the NIMP of a DDI study

Decision 1

\begin{tabular}{lll}
\hline Grade (severity) & Seriousness & $\begin{array}{l}\text { Individuals } \\
\text { Action }\end{array}$
\end{tabular}

I (Mild) N/A No action required - UNLESS: Presence of clinically relevant signs and symptoms of magnesium toxicity with confirmed hypermagnesaemia on clinical laboratory tests, in which case the subject will not receive any urther doses of $\mathrm{MgSO} 4$ or $\mathrm{MgSO} 4 / \mathrm{IMP}$ and will consequently be withdrawn from the study.

II (Moderate) N/A

III (Severe) Not serious

III (Severe) $\quad$ Serious (all except life-threatening and fatal)

IV (life-threatening) Serious (life-threatening but non-fatal)

V (fatal) Serious (fatal only)
IMP administration may be continued, amended, temporarily suspended or discontinued in accordance with investigator's clinical judgement and relevant algorithms for the treatment of toxicities.

IMP administration will be discontinued. Affected IMP/NIMP administration will be discontinued; dosing with remaining IMP/NIMPs can continue in accordance with

Adaptive Features and their limits, Investigator's clinical judgement and relevant algorithms for the treatment of toxicities.

IMP administration will be discontinued.

IMP administration will be discontinued.

N/A 
Within-cohort (decision 2) These needed to exempt expected ARs where their appearance would imply efficacy. The numberbased approach to stopping after a certain amount of grade 2 or 3 ARs was not necessary, as the drug had an SPC and the frequency of ARs already understood - this trial was not looking to identify a potential unknown safety signal. The rules instead needed to focus on when to stop the whole cohort and this could be done based on the number of individual withdrawals. It was important to ensure that the lower dose arm could continue if the higher dose arm had to stop-i.e. the option top unblind was built in to allow the affected dose arm to be identified (Table 3 ).

Study progression (decision 3) Not required (no dose escalation decisions or other study parts).

The rules thus facilitated study continuation by allowing decisions to be made after unblinding (thereby potentially allowing the low dose arm to continue) and by ensuring that presentations to emergency departments and possible admission to hospital with no significant intervention did not immediately result in suspension.

\section{Example 3}

A phase 1 ethnic comparator study used a single cohort of Japanese subjects to compare the safety and tolerability of an IMP (that often had off-target ARs) against that of Caucasian subjects. The IB listed several gastrointestinal ARs (including vomiting and diarrhoea) from earlier phase 1 studies, which often reduced severity with continued dosing.

Individual rules (decision 1) For unexpected ARs, the template individual AR rules were used, but for the anticipated gastrointestinal ARs, stricter rules based on duration were devised (Table 4).
Within-cohort (decision 2) Template rules for serious ARs; non-serious AR rules simply based on number of withdrawals.

Study progression (decision 3) Not required.

\section{Modifications for anticipated effects based on class effect or mode of action}

Drugs in their first year of clinical development may have no or very limited safety information in the IB. However, the mode of action or non-clinical toxicity data may predict drug effects in humans, including potentially serious adverse effects. It is advisable to modify the rules so that they cater for the worst-case scenario for fundamental risks. Ensure that there are multiple options available for other, lower risk scenarios so that the study is not prematurely suspended, e.g. for non-severe, non-serious predicted drug effects.

Additionally, class effects or non-clinical toxicity data may indicate potential ARs that do not necessarily pose a significant risk to volunteers and may indicate drug efficacy. In line with the FDA guidance [11], the rules should distinguish between the anticipated ARs that are acceptable (i.e. ARs that, if observed within specified parameters, will not require changes to the ongoing and planned dosing regimens) and those that are unacceptable. The rules should contain a clear list of anticipated ARs that are acceptable and exempt them from the rules used for all unanticipated/unacceptable ARs.

\section{Example}

New investigational drugs intended for analgesia may be expected to produce opioid-related side effects if opioid receptors or subtypes are potential targets. Potential ARs
Table 3 Within-cohort rules for anticipated ARs, based on the SPC of the IMP

\begin{tabular}{|c|c|}
\hline Severity & $\begin{array}{l}\text { Decision } 2 \\
\text { Actions }\end{array}$ \\
\hline Rules for serious ARs & $\begin{array}{l}\text { If the SC decides not to unblind, the whole study will be suspended if } 2 \text { or more } \\
\text { subjects experience serious expected ARs. If the SC decides to unblind, the } \\
\text { following rule applies: } \\
\text { The high dose group only will be suspended (and the low dose group allowed to } \\
\text { continue) if both (or all) serious expected ARs occur only in the high dose group. } \\
\text { * Note: Due to the nature and intended indication of this compound, it is possible } \\
\text { that subjects who experience these ARs [listed; omitted here for space] may be } \\
\text { hospitalised for investigation and minor intervention. In this situation, the above } \\
\text { rules apply. However, if major intervention occurs, only } 1 \text { serious expected AR } \\
\text { is required to terminate the study (or the higher dose group only, if unblinding } \\
\text { reveals the AR to have occurred in this group). }\end{array}$ \\
\hline $\begin{array}{l}\text { Rules for severe, } \\
\text { non-serious ARs }\end{array}$ & $\begin{array}{l}\text { If the SC decides not to unblind, the whole study will be suspended if } 3 \text { or more } \\
\text { subjects experience severe, non-serious expected ARs. If the SC decides to } \\
\text { unblind, the following rule applies: } \\
\text { The higher dose group only will be suspended (and the lower dose group is allowed } \\
\text { to continue) if } 3 \text { severe, non-serious expected ARs occur. }\end{array}$ \\
\hline
\end{tabular}


Table 4 Individual AR rules for anticipated effects based on safety data in the IB

\begin{tabular}{|c|c|c|c|}
\hline Adverse event & CTCAE grading & Duration of event & $\begin{array}{l}\text { Decision } 1 \\
\text { Individuals-action: }\end{array}$ \\
\hline \multirow[t]{4}{*}{ Vomiting } & Grade 1 & N/A & No action required \\
\hline & Grade 2 & $\leq 24 \mathrm{~h}$ & $\begin{array}{l}\text { IMP administration may be continued or discontinued in accordance with Investigator's } \\
\text { clinical judgement and relevant algorithms for the treatment of clinically significant } \\
\text { adverse events (at least possibly IMP related). }\end{array}$ \\
\hline & & $>24 \mathrm{~h}$ & Discontinue dosing and withdraw from trial \\
\hline & $\geq$ Grade 3 & N/A & Discontinue dosing and withdraw from trial \\
\hline \multirow[t]{5}{*}{ Diarrhoea } & Grade 1 & N/A & No action required \\
\hline & Grade 2 & $\leq 72 \mathrm{~h}$ & $\begin{array}{l}\text { IMP administration may be continued or discontinued in accordance with Investigator's } \\
\text { clinical judgement and relevant algorithms for the treatment of clinically significant } \\
\text { adverse events (at least possibly IMP related). }\end{array}$ \\
\hline & & $>72 \mathrm{~h}$ & Discontinue dosing and withdraw from trial \\
\hline & $\geq$ Grade 3 & $\leq 48 \mathrm{~h}$ & $\begin{array}{l}\text { IMP administration may be continued or discontinued in accordance with Investigator's } \\
\text { clinical judgement and relevant algorithms for the treatment of clinically significant } \\
\text { adverse events (at least possibly IMP related). }\end{array}$ \\
\hline & & $>48 \mathrm{~h}$ & Discontinue dosing and withdraw from trial \\
\hline
\end{tabular}

may be anticipated based on class effects of marketed opioids and it is advisable to use a strong opioid (e.g. oxycodone) as a worst-case scenario. Opioids have welldocumented undesirable effects, some of which are common to many opioids and their appearance would indicate on-target action. Thus, stopping a trial because of their occurrence would be counter-productive, especially where the ARs are unlikely to place subjects under supervision in a trials unit at excessive risk (e.g. nausea, dizziness, headache, constipation). Other opioid-related undesirable effects (e.g. confusion, dyspnoea) would not be acceptable in healthy volunteers at any CTCAE grade and would likely prevent further dosing. In this situation, it is advisable to use the SPC of the most appropriate marketed product as a reference and identify which ARs would be acceptable and which would be unacceptable [11].

Using oxycodone as an example of a reference product, its SPC [17] has an extensive list of undesirable effects, so consider focussing on those that are 'very common' (frequency $\geq$ $1 / 10$ ) and 'common' (frequency $\geq 1 / 100$ to $<1 / 10$ ). The acceptable anticipated ARs at this frequency should be identified, listed clearly in the protocol and exempted from the template AR rules. Additionally, no AR would be acceptable unconditionally and a limit on severity would also be necessary. Undesirable effects in the SPC that are uncommon or rare may not emerge in a clinical trial with a comparatively smaller sample size than the general patient population, so for the purposes of a clinical trial, they may be regarded as 'unanticipated'.

For all unacceptable, anticipated ARs, as well as all other unanticipated ARs, the template AR rules would apply.
Table 5 shows an example of how the anticipated ARs were identified based on the common/very common undesirable effects of oxycodone's SPC and separated into acceptable and unacceptable. This approach has served as a template for early phase trials conducted in potential new analgesics.

\section{Modification to allow more grade 2 SAEs}

Grade 2 ARs are often assumed to be all non-serious. This may not always be the case, as the threshold at which people present to healthcare is not consistently at the same level of severity. Patients may be admitted for logistical reasons, lack of senior staff or investigations (especially out-of-hours) but receive no significant investigation or treatment. Consequently, such an AR may have resulted in hospitalisation (and therefore fulfil SAE criteria) but in clinical terms not exceed grade 2. Withdrawing an individual or stopping a cohort/study for one grade 2 serious AR may thus be unnecessary or counter-productive. Consider treating SAEs limited to grade 2 as per grade 3, non-serious ARs. This approach has been used in first-in-human and other early phase trials, where the amended template AR rules tables (Table 6) were approved by the UK competent authorities.

Alternatively, the definition of 'hospitalisation' for the purposes of SAE designation could be clarified in protocol. Admissions for logistical reasons, minor investigation or non-urgent treatment, or where no abnormalities were identified and no intervention was needed, could be excluded from the definition of hospitalisation for purposes of SAE reporting, the rationale being that no secondary care was clinically required. Without refining the hospitalisation definition or adapting the AR rules, a 
Table 5 Acceptable and unacceptable anticipated ARs, based on common and very common ARs listed in the oxycodone spc [20]

\begin{tabular}{lll}
\hline Unacceptable & \multicolumn{1}{l}{ Acceptable } & \\
\cline { 2 - 3 } (Template AR rules apply) & (Exempt from template AR rules at CTCAE grades 1 and 2 \\
\cline { 2 - 3 } Common & Very common & Common \\
\hline Anxiety & Somnolence & Decreased appetite \\
Confusional state & Dizziness & Insomnia \\
Depression & Headache & Nervousness \\
Abnormal thinking & Constipation & Abnormal dreams \\
Sedation & Nausea & Tremor \\
Dyspnoea & Vomiting & Lethargy \\
Bronchospasm & Pruritus & Abdominal pain \\
& & Diarrhoea \\
& & Dry mouth \\
& & Dyspepsia \\
& & Rash \\
& & Hyperhidrosis \\
& & Asthenia \\
& & Fatigue \\
& & Cough decreased \\
& &
\end{tabular}

single volunteer with an $\mathrm{AE}$ at moderate severity has the potential to cause whole trial termination due to anxiety regarding the $\mathrm{AE}$ or lack of availability to non-urgent care services.

\section{Modifications for different routes of administration}

For trials using formulations of an IMP with different routes of administration (e.g. subcutaneous, intravenous, oral), the rules

Table 6 Modified template rules to allow continuation with one grade 2 SAR

\begin{tabular}{|c|c|c|c|c|c|c|c|}
\hline \multirow[b]{3}{*}{$\begin{array}{c}\text { Grade } \\
\text { (Severity) }\end{array}$} & \multirow[b]{3}{*}{ Seriousness } & \multirow{3}{*}{$\begin{array}{c}\text { DECISION } 1 \\
\text { Individuals } \\
\text { Action }\end{array}$} & \multicolumn{4}{|r|}{ DECISION 2} & \multirow{3}{*}{$\begin{array}{c}\text { DECISION } 3 \\
\text { Escalation and/or progression } \\
\text { Action }\end{array}$} \\
\hline & & & \multicolumn{2}{|c|}{$\begin{array}{l}\text { Number of } \\
\text { subjects }\end{array}$} & \multirow{2}{*}{$\begin{array}{l}\text { Showing } \\
\text { signs of } \\
\text { reversibility } \\
\text { within [state } \\
\text { time period] }\end{array}$} & Continuation within a dosing regimen & \\
\hline & & & $\begin{array}{l}\text { In } \\
\text { one } \\
\text { soc }\end{array}$ & $\underset{\text { total }}{\ln }$ & & $\begin{array}{l}\text { Action } \\
\text { NOTE 1: In the event of suspension, continuation or } \\
\text { extension of the cohort requires a substantial amendment. }\end{array}$ & \\
\hline (Mild) & $\mathrm{N} / \mathrm{A}$ & N/A & Any & Any & $\mathrm{N} / \mathrm{A}$ & No action required & No action required (Action $\mathrm{A}$ ). \\
\hline \multirow[t]{6}{*}{ (Moderate) } & \multirow[t]{4}{*}{ Not serious } & \multirow{4}{*}{$\begin{array}{l}\text { IMP administration may } \\
\text { be continued, amended, } \\
\text { temporarily suspended } \\
\text { or discontinued in } \\
\text { accordance with } \\
\text { investigator's clinical } \\
\text { judgement and relevant } \\
\text { algorithms for the } \\
\text { treatment of toxicities. }\end{array}$} & $\leq 2$ & $\leq 3$ & Yes & $\begin{array}{l}\text { Dosing of the remainder of the dosing regimen can } \\
\text { continue as per CSP. Dosing regimen can be } \\
\text { extended. }\end{array}$ & Action (B) \\
\hline & & & $\geq 3$ & $\geq 4$ & Yes & $\begin{array}{l}\text { Dosing of the remainder of the dosing regimen } \\
\text { suspended. }\end{array}$ & Action (C) \\
\hline & & & \multirow[t]{2}{*}{$\mathrm{N} / \mathrm{A}$} & 1 & No & $\begin{array}{l}\text { Dosing of the remainder of the dosing regimen can } \\
\text { continue as per CSP. Dosing regimen can be } \\
\text { extended. }\end{array}$ & Action (B) \\
\hline & & & & $\geq 2$ & No & $\begin{array}{l}\text { Dosing of the remainder of the dosing regimen } \\
\text { suspended. }\end{array}$ & Action (C) \\
\hline & \multirow[t]{2}{*}{ Serious } & \multirow[t]{2}{*}{$\begin{array}{l}\text { IMP administration will } \\
\text { be discontinued. }\end{array}$} & N/A & 1 & $\mathrm{~N} / \mathrm{A}$ & $\begin{array}{l}\text { Dosing of the remainder of the dosing regimen can } \\
\text { continue as per CSP. Dosing regimen can be } \\
\text { extended. }\end{array}$ & Action (B) \\
\hline & & & N/A & 2 & $\mathrm{~N} / \mathrm{A}$ & $\begin{array}{l}\text { Dosing of the remainder of the dosing regimen } \\
\text { suspended. }\end{array}$ & Action $(\mathrm{C})$ \\
\hline \multirow[t]{4}{*}{$\begin{array}{l}\text { III } \\
\text { (Severe) }\end{array}$} & \multirow[t]{3}{*}{ Not serious } & \multirow[t]{3}{*}{$\begin{array}{l}\text { IMP administration will } \\
\text { be discontinued. }\end{array}$} & \multirow[t]{3}{*}{ N/A } & 1 & Yes & $\begin{array}{l}\text { Dosing of the remainder of the dosina reaimen can } \\
\text { continue as per CSP. Dosing regimen can be } \\
\text { extended. }\end{array}$ & Action (B) \\
\hline & & & & $\geq 2$ & Yes & \multirow{5}{*}{$\begin{array}{l}\text { Dosing of the remainder of the dosing regimen } \\
\text { suspended. }\end{array}$} & \multirow[b]{3}{*}{ Action (C) } \\
\hline & & & & $\geq 1$ & No & & \\
\hline & $\begin{array}{l}\text { Serious (all } \\
\text { except life- } \\
\text { threatening } \\
\text { and fatal) }\end{array}$ & $\begin{array}{l}\text { IMP administration will } \\
\text { be discontinued. }\end{array}$ & $\mathrm{N} / \mathrm{A}$ & $\geq 1$ & $\mathrm{~N} / \mathrm{A}$ & & \\
\hline $\begin{array}{l}\text { IV } \\
\text { (life- } \\
\text { threatening) }\end{array}$ & $\begin{array}{l}\text { Serious (life- } \\
\text { threatening } \\
\text { but non-fatal) }\end{array}$ & $\begin{array}{l}\text { IMP administration will } \\
\text { be discontinued. }\end{array}$ & $\mathrm{N} / \mathrm{A}$ & $\geq 1$ & $\mathrm{~N} / \mathrm{A}$ & & \multirow[t]{2}{*}{ Study suspended, Action (D) } \\
\hline $\begin{array}{l}\text { V } \\
\text { (fatal) }\end{array}$ & $\begin{array}{l}\text { Serious (fatal } \\
\text { only) }\end{array}$ & $\mathrm{N} / \mathrm{A}$ & $\mathrm{N} / \mathrm{A}$ & $\geq 1$ & $\mathrm{~N} / \mathrm{A}$ & & \\
\hline
\end{tabular}


can be adapted to ensure that ARs relating to an administration site do not necessarily impact on other cohorts with a different route of administration.

\section{Example}

For trials using an IMP with subcutaneous (SC) and intravenous (IV) administration routes, the rules were adapted to ensure that ARs related to the IMP covered both treatment arms in parallel, but SC injection-site reactions and IV infusion-related reactions did not prevent continuation of the other arm. The latter may be particularly important where the assessment of pharmacokinetics from one particular formulation is a primary objective.

Individual rules (decision 1) Template rules used.

Within-cohort (decision 2) Modified, see Table 7.

Study progression (decision 3) Modified, see Table 7.

\section{Modifications to AR rules in response to emerging human data}

With the best will in the world, the rules designed prior to any human being dosed (and therefore based on non-clinical data) are an estimation of what may or may not occur and therefore have to be very cautious. Emerging human data may show that rules devised prior to initiation of human trials are unnecessarily strict and may need amendment to allow the drug development programme to continue.
Human data may show diagnosis-based rules to be inappropriate and an assessment of the overall clinical picture may be more appropriate. When amending AR rules, the aim is to set the boundary between acceptable and unacceptable levels of symptoms/signs.

\section{Example}

Trials in immunology compounds can have specific challenges when designing AR rules for anticipated effects. Compounds that have immune-stimulatory effects (e.g. anticancer drugs) may produce multiple, similar ARs such as flulike symptoms and cytokine release syndrome, caused by the IMP-induced release of cytokines. The potentially lifethreatening cytokine release syndrome may not be anticipated, but it is theoretically possible and the worst-case scenario that would not be acceptable in healthy volunteers in a first-inhuman trial. Therefore, very cautious rules specifically for these signs/symptoms should be considered. Other local and systemic immune-stimulatory effects (flu-like symptoms and injection site reactions) may be anticipated but, not being lifethreatening and their occurrence indicating drug efficacy, separate, less cautious rules may be suitable. An example of potential individual rules shown in Table 8 . These rules were designed prior to trial initiation in a first-in-human trial in an investigational immune-stimulatory anti-cancer treatment administered subcutaneously and based on what was anticipated from pre-clinical data or mode of action. To minimise risk, they have been tightened to withdraw subjects at grade 1 for cytokine release syndrome, but not for isolated symptoms of cytokine release, e.g. flu-like symptoms. For all other, unanticipated ARs, the template rules can be used.

Table 7 Within-cohort and study progression AR rules for different administration routes

\begin{tabular}{|c|c|c|c|}
\hline $\begin{array}{l}\text { Grade } \\
\text { (severity) }\end{array}$ & Subjects affected & $\begin{array}{l}\text { Decision } 2 \\
\text { Action }\end{array}$ & $\begin{array}{l}\text { Decision } 3 \\
\text { Effect on cohort progression }\end{array}$ \\
\hline I (Mild) & $\begin{array}{l}\text { N/A } \\
\leq 2 \text { subjects in different } \mathrm{SOC}\end{array}$ & Dose regimen may continue & Study continues as per clinical study protocol \\
\hline \multirow[t]{2}{*}{ II (Moderate) } & $\begin{array}{c}2 \text { subjects in same SOC OR } 3 \\
\text { subjects in different SOC }\end{array}$ & & $\begin{array}{l}\text { Successive cohorts may commence, } \\
\text { if they have not already }\end{array}$ \\
\hline & $\begin{array}{c}\geq 3 \text { subjects in same SOC or } \geq 4 \\
\text { subjects in different SOC }\end{array}$ & $\begin{array}{l}\text { All dose regimens suspended UNLESS } \\
\text { the AR is either a local tolerability event } \\
\text { or and injection/infusion site reaction, in } \\
\text { which case only the affected cohort is } \\
\text { suspended }\end{array}$ & $\begin{array}{l}\text { Study continuation requires substantial } \\
\text { amendment. Continuation } \\
\text { of the affected cohort requires } \\
\text { substantial amendment }\end{array}$ \\
\hline \multirow{2}{*}{$\begin{array}{l}\text { III (Severe, } \\
\text { not } \\
\text { serious) }\end{array}$} & 1 subject & Dose regimen may continue & $\begin{array}{l}\text { Study continues as per clinical study } \\
\text { protocol }\end{array}$ \\
\hline & $\geq 2$ subjects in same $\mathrm{SOC}$ or & $\begin{array}{l}\text { All dose regimens suspended UNLESS the AR is } \\
\text { either a local tolerability event or and } \\
\text { injection/infusion site reaction, in which case } \\
\text { only the affected cohort is suspended }\end{array}$ & $\begin{array}{l}\text { Study continuation requires substantial } \\
\text { amendment. Continuation of the affected } \\
\text { cohort requires substantial amendment }\end{array}$ \\
\hline
\end{tabular}


Table 8 Individual rules devised prior to study initiation, based on anticipated effects

\begin{tabular}{llll}
\hline Grade (severity) & Diagnosis & Reversibility & $\begin{array}{l}\text { Decision } 1 \\
\text { Action }\end{array}$ \\
\hline I (Mild) & Cytokine release syndrome & N/A & IMP administration will be discontinued \\
& Flu-like symptoms & N/A & No action required \\
& Injection site reactions & N/A & No action required \\
& All other toxicities & N/A & No action required \\
Cytokine release syndrome & N/A & IMP administration will be discontinued \\
& Flu-like symptoms & N/A & IMP administration will be discontinued \\
& Injection site reaction & N/A & No action required \\
& All other toxicities & Yes & IMP administration may be continued, amended, temporarily \\
& & & suspended or discontinued in accordance with Investigator's \\
& & No linical judgement and relevant algorithms for the treatment of toxicities
\end{tabular}

Upon trial initiation, human data may provide evidence of what ARs arise in humans and diagnoses that have similar signs and symptoms may not be readily distinguishable, especially at lower CTCAE grades. The two closely related diagnoses (flu-like symptoms and cytokine release syndrome) may be scientifically distinguishable but not necessarily so clinically and the transition from desirable pharmacodynamic effect to unacceptable safety risk may be unclear. In this case, the existing AR rules risk leading to premature trial termination.

Consequently, amended AR rules should look at the overall clinical picture seen in human subjects, taking into account all signs, symptoms and laboratory findings and not try to distinguish between specific diagnoses if this cannot reliably be done. The amended rules should instead set the threshold at which individuals and cohorts should be withdrawn from dosing more appropriately. Amended rules should be presented to the competent authority (along with relevant human safety and pharmacodynamic data as a justification) for approval. Any amendment to the rules in the protocol approved by the competent authorities will likely be substantial. Changes to make them less strict have the potential to expose more subjects to ARs, while changes to make the rules stricter may reflect a change in the risk profile of the IMP. For both these scenarios, competent authority approval should be sought. This approach has been used in early phase clinical trials and the amended rules, with sufficient justification, were subsequently approved.

\section{Limitations}

Algorithms such as the template AR rules presented here are useful in ensuring a consistent approach to dealing with ARs. However, such algorithms have limitations. Appropriate, safe use relies on accurate clinical assessment, diagnosis and grading.
For individual subject safety, a more cautious clinical judgement by an experienced physician should be allowed to override an approved algorithm that is seen as less cautious under the circumstances. However, the opposite does not apply: competent authority-approved algorithms designed to safeguard participants should not be overridden by a physician if the algorithm is more cautious than their clinical judgement.

Finally, the template rules are an algorithm to guide decisions on dosing with the IMP. It is not a tool that can replace sound clinical judgement, nor is it a tool that can guide clinical management of affected individuals.

\section{Conclusion}

Stopping (AR) rules in early phase clinical trials are a regulatory requirement and necessary for subject safety. However, designing AR rules can present challenges. Firstly, they need to ensure that trials do not get terminated prematurely without clinical justification. Secondly, they may need to accommodate multiple concurrent trial arms or parts and, thirdly, where IMPs have anticipated ARs based on class effect, mode of action or pre-clinical data, the boundary between acceptable and unacceptable ARs needs to be determined. To address these challenges, this manuscript presents a one-page template AR rules table that can be used for all trial designs, including large integrated trials. It can be adapted for specific IMPs or trials if necessary, taking into account anticipated effects. It fulfils regulatory requirements and has been tried and tested, having been approved and used for many trials in the UK. This template approach ensures the assessment of ARs and the application of AR rules is systematic, objective and consistent.

Authors' contributions All authors participated in the study design, data collection and analysis of the data. All authors reviewed and approved the manuscript. 


\section{Compliance with ethical standards}

Conflict of interest The authors declare that they have no conflicts of interest.

OpenAccess This article is distributed under the terms of the Creative Commons Attribution 4.0 International License (http:// creativecommons.org/licenses/by/4.0/), which permits unrestricted use, distribution, and reproduction in any medium, provided you give appropriate credit to the original author(s) and the source, provide a link to the Creative Commons license, and indicate if changes were made.

Publisher's Note Springer Nature remains neutral with regard to jurisdictional claims in published maps and institutional affiliations.?

\section{References}

1. Directive 2001/20/EC of the European Parliament and of the Council of 4 April 2001. (2001) https://ec.europa.eu/health/sites/ health/files/files/eudralex/vol-1/dir_2001_20/dir_2001_20_en.pdf. Accessed 31 August 2018

2. Regulation (EU) No 536/2014 of the European Parliament and the Council of 16 April 2014 on clinical trials on medicinal products for human use, and repealing Directive 2001/20/EC. (2014) https:/ec. europa.eu/health/sites/health/files/files/eudralex/vol-1/reg_2014 536/reg_2014_536_en.pdf. Accessed 31 August 2018

3. Guideline for Good Clinical Practice E6(R2). (2016) https://www. ich.org/fileadmin/Public_Web_Site/ICH_Products/Guidelines/ Efficacy/E6/E6_R2_Step_4_2016_1109.pdf. Accessed 31 August 2018

4. Guideline on strategies to identify and mitigate risks for first-inhuman and early clinical trials with investigational medicinal products. (2016) http://www.ema.europa.eu/docs/en_GB/document library/Scientific_guideline/2017/07/WC500232186.pdf. Accessed 31 August 2018

5. Clinical Trial Facilitation Group Q\&A document - Reference Safety Information. (2017) http://www.hma.eu/fileadmin/dateien/ Human_Medicines/01-About_HMA/Working_Groups/CTFG/ 2017_11_CTFG_Question_and_Answer_on_Reference_Safety_ Information_2017. pdf. Accessed 31 August 2018

6. Eddleston M, Cohen AF, Webb DJ (2016) Implications of the BIA102474-101 study for review of first-into-human clinical trials. Br J Clin Pharmacol 81(4):582-586
7. Kaur R, Sidhu P, Singh S (2016) What failed BIA 10-2474 phase I clinical trial? Global speculations and recommendations for future phase I trials. J Pharmacol Pharmacother 7(3):120-126

8. H Attarwala H (2017) TGN1412: from discovery to disaster. J Young Pharm 2(3):332-336

9. Fruhner K, Hartmann G, Sudhop T (2017) Analysis of integrated clinical trial protocols in early phases of medicinal product development. Eur J Clin Pharmacol 73(12):1565-1577

10. Tyson JE, Pedroza C, Wallace D, D'Angio C, Bell EF, Das A (2016) Stopping guidelines for an effectiveness trial: what should the protocol specify? Trials 17:240

11. Good Review Practice: clinical review of investigational new drug applications. (2013) https://www.fda.gov/downloads/Drugs/ GuidanceComplianceRegulatoryInformation/UCM377108.pdf. Accessed 05 September 2018

12. Lorch U, Berelowitz K, Ozen C, Naseem A, Akuffo E, Taubel J (2012) The practical application of adaptive study design in early phase clinical trials: a retrospective analysis of time savings. Eur J Clin Pharmacol 68(5):543-551

13. Lorch U, O'Kane M, Taubel J (2014) Three steps to writing adaptive study protocols in the early phase clinical development of new medicines. BMC Med Res Methodol 14:84

14. Common Terminology Criteria for Adverse Events (CTCAE) version 5.0. (2017) https://ctep.cancer.gov/protocolDevelopment/ electronic_applications/docs/CTCAE_v5_Quick_Reference_5x7. pdf. Accessed 31 August 2018

15. Toxicity Grading Scale for Healthy Adult and Adolescent Volunteers Enrolled in Preventive Vaccine Clinical Trials (2007). https://www.fda.gov/downloads/BiologicsBloodVaccines/ ucm091977. Accessed 31 August 2018

16. Division of AIDS (DAIDS) Table for Grading the Severity of Adult and Pediatric Adverse Events. Version 2.0. (2014) https://rsc.techres.com/docs/default-source/safety/daids_ae_grading_table_v2 nov2014.pdf. Accessed 31 August 2018

17. Understanding MedDRA The Medical Dictionary for Regulatory Activities (2013) https://www.meddra.org/sites/default/files/page/ documents/meddra2013.pdf. Accessed 31 August 2018

18. Coates S, Täubel J and Lorch U (2017). Practical risk management in early phase clinical trials. Front. Pharmacol. Conference Abstract: EUFEMED 2017. https://doi.org/10.3389/conf.fphar. 2017.62.00014

19. Coates S, Taubel J, Bowen J, Lorch U. Practical risk management for adaptive integrated early phase clinical trials https://www. micenavi.jp/wcp2018/search/detail program/id:1448. Last accessed 29/10/2018

20. Oxynorm $5 \mathrm{mg} \mathrm{SPC}$. https://www.medicines.org.uk/emc/product/ 3850/smpc. Accessed 05 September 2018 